\title{
UTILITY FUNCTIONS WHICH ENSURE THE ADEQUACY OF STATIONARY STRATEGIES
}

\author{
MICHAEL G. MONTICINO
}

\begin{abstract}
Within a Dubins and Savage gambling framework, a stationary strategy is a strategy which selects a gamble at each time based solely on the gambler's present fortune. We determine conditions upon the gambler's utility function under which stationary strategies allow the gambler to maximize his return. The class of utility functions which satisfies these conditions, termed nearly leavable shift invariant functions, is large and contains many of the common gambling utility functions. Moreover, this class is closed under uniform limits. These results are obtained with the setting of an analytic gambling house.
\end{abstract}

\section{INTRODUCTION}

Quite naturally a gambler would like to maximize his winnings while employing as simple a gambling scheme as possible. In this paper, within a Dubins and Savage gambling framework, we determine conditions under which a gambler may approach this goal. Specifically, we establish conditions upon the gambler's utility function which ensure that stationary strategies are adequate to enable the gambler to maximize his return. The class of functions which satisfies these conditions is large and includes many of the common gambling utility functions. A stationary strategy is uncomplicated in the sense that it is a plan which tells a gambler how to wager when he has a particular fortune without regard to his past financial history.

Informally, a gambling strategy is a plan telling a player how to gamble when he is at a certain state and has experienced a particular partial history. A gamble is a probability measure on a set, $F$, called the state space. The value, $\gamma$, that a gamble assigns to a subset of the state space is the probability that the gambler will move to a state within that subset if he elects to use $\gamma$. If a gambler has experienced a partial history $\left(x_{1}, x_{2}, \ldots, x_{n}\right)$ this means that on day one he was at state $x_{1}$, on day two he was at state $x_{2}$ and so on. To be more formal, a partial history is a finite sequence of elements of $F$, and a strategy is a gamble valued function defined on the set of partial histories. The history space, $H$, is the set of infinite day histories, that is, $H=F \times F \times F \times \cdots$. If a gambler has experienced partial history $\left(x_{1}, x_{2}, \ldots, x_{n}\right)$ and he has elected to use a

Received by the editors October 17, 1988 and, in revised form, April 13, 1989.

1980 Mathematics Subject Classification (1985 Revision). Primary 60G40, 62L15.

Key words and phrases. Gambling, optimal return, stationary strategies, utility function. 
strategy $\sigma$, then on day $n$, when he is at state $x_{n}$, his next move would be to use the gamble $\sigma\left(x_{1}, \ldots, x_{n}\right)$. A strategy, $\sigma$, is stationary if for all $n$ and all $\left(x_{1}, \ldots, x_{n}\right)$, the gamble selected by $\sigma$ at $\left(x_{1}, \ldots, x_{n}\right)$ is dependent only on the value of $x_{n}$.

$V(x)$ is the optimal return at a point, $x$, in $F$. It is the best the gambler can hope to accomplish towards reaching some given objective or goal while selecting from strategies available to him at $x$. A real valued function, $g$, termed the utility function, is defined on $H$. The utility of a strategy, $\sigma$, is defined to be the integral of $g$ with respect to the measure determined by $\sigma$ (these concepts will be made precise in the next section). For example, $g$ could give the amount of money that gambler has on a particular day. And the utility of $\sigma$ would be the gambler's expected fortune on that day, if the strategy $\sigma$ was used. The value of $V(x)$, then, is the supremum of the utilities of all strategies available at $x$. Obviously, a gambler starting at $x$ desires to select a strategy available to him whose utility is close to being optimal, that is, close to $V(x)$. The question we are concerned with is what are necessary and sufficient conditions upon the utility function such that the gambler may do this by selecting some stationary strategy.

In [19] it was shown, under certain conditions on the gambling house, that stationary strategies are adequate to allow the gambler to maximize his return if

$$
g(h)=u^{*}(h)=\limsup _{n \rightarrow \infty} u\left(x_{n}\right)
$$

for real valued $u$. Then in [17] it was demonstrated that stationary strategies are adequate if $F$ is finite and

$$
g(h)=u_{*}(h)=\liminf _{n \rightarrow \infty} u\left(x_{n}\right) .
$$

However, an example in [17] showed that the set of utility functions for which stationary strategies are adequate is not closed under addition.

We extend these results and show that for a broad class of utility functions, termed nearly leavable shift invariant functions, stationary strategies are adequate for approximating optimal return. This class of functions includes the classic Dubins and Savage utility function, $g(h)=\limsup _{n \rightarrow \infty} u\left(x_{n}\right)$. It also includes those studied by Sudderth in [17]. Moreover, we show that this class of utility functions is closed under uniform limits.

Previous work has concentrated upon characterizing the types of gambling houses for which stationary strategies are adequate for the utility function $g(h)=\limsup _{n \rightarrow \infty} u\left(x_{n}\right)$. For instance, in [6] the adequacy of stationary strategies was verified in the case that the state space was finite, and the gambling house was leavable. A gambling house is leavable if the gambler can effectively stop gambling at any time and leave with his current fortune. Ornstein, in [13], demonstrated that in the countable state space case, if the gambles available were countably additive, then the above result still held. In fact, he proved a stronger result, that stationary plans are uniformly adequate (definitions given 
in the next section). However, an example showed that if $F$ is not countable then stationary plans need not be uniformly adequate. The state space in the example had cardinality greater than that of the continuum. It was later shown, in [7], that there exists a leavable gambling house, defined with a standard Borel space as the state space, for which stationary plans are not uniformly adequate, if noncountably additive gambles are allowed. The question of uniform adequacy of stationary plans in leavable gambling problems, for which the state space is Borel and only countable additive gambles are allowed, has remained open until recently. Blackwell and Ramakrishnan in [3] have demonstrated that there exist leavable Borel gambling problems for which stationary plans are not uniformly adequate.

Analogous work has been done in the field of Markov strategies. A strategy, $\sigma$, is Markov if there exists a sequence, $\gamma_{1}, \gamma_{2}, \ldots$, of gamble valued functions such that for each $n$ and $\left(x_{1}, \ldots, x_{n}\right), \sigma\left(x_{1}, \ldots, x_{n}\right)=\gamma_{n}\left(x_{n}\right)$. Hill in [9] showed that if $F$ is finite, then for a general class of utility functions, called shift and permutation invariant, Markov strategies are adequate for approximating optimal return. Hill and Pestien [10] presented another class of utility functions, which included the total reward, the average reward, and the periodic reward functions, for which Markov strategies are adequate.

In $\S 2$ of this paper, we present necessary definitions and terminology. We also formally state our main result, reserving the proof until $\S 5$. The basic gambling concepts are as introduced by Dubins and Savage in [6]. We characterize the class of nearly leavable shift invariant functions in $\S 3$. Technical results, which are necessary to prove our main theorem, are presented in $\S 4$. In $\S 5$ we prove this theorem-that is, it is sufficient for the utility function to be nearly leavable shift invariant for stationary strategies to be adequate for approximating optimal return. The necessity of this condition is discussed in $\S 6$.

\section{Preliminaries}

Here a gambling problem is a triple $(F, \Gamma, g)$, where

(i) the state space, $F$, is a nonempty set, the set of partial histories is the set $\bigcup_{n} F^{n}$, and the space of histories, $H$, is the countably infinite product $F \times F \times \cdots$;

(ii) the gambling house, $\Gamma$, is a subset of $F \times P(F)$, where $P(F)$ is the set of finitely additive probability measures defined on all subsets of $F$; for $x \in F, \Gamma(x)=\{\gamma:(x, \gamma) \in \Gamma\}$ is the set of gambles available at $x ;$ and

(iii) the utility function, $g$, is a real valued function defined on $H$.

As mentioned, a strategy can be defined as a gamble valued function on the set of partial histories. However, it is convenient for notational reasons to use the following equivalent definition. A strategy, $\sigma$, is a sequence $\sigma_{0}, \sigma_{1}, \ldots$, where $\sigma_{0} \in P(F)$ and for each $n, \sigma_{n}$ is a map from $F^{n}$ into $P(F)$. A strategy is available at $x \in F$ if $\sigma_{0} \in \Gamma(x)$, and for each $n$ and $\left(x_{1}, \ldots, x_{n}\right) \in F^{n}$, 
$\sigma_{n}\left(x_{1}, \ldots, x_{n}\right) \in \Gamma\left(x_{n}\right) . \Sigma(x)$ will denote the set of all strategies available at $x$.

If $p=\left(x_{1}, \ldots, x_{n}\right) \in F^{n}$, then $\sigma[p]$ denotes the conditional strategy given $p$, which is defined by

$$
(\sigma[p])_{0}=\sigma_{n}\left(x_{1}, \ldots, x_{n}\right)
$$

and for $k>0$

$$
(\sigma[p])_{k}\left(y_{1}, \ldots, y_{k}\right)=\sigma_{n+k}\left(x_{1}, \ldots, x_{n}, y_{1}, \ldots, y_{k}\right) .
$$

$H$ is endowed with the product topology determined by giving $F$ the discrete topology. A function $g: H \rightarrow \mathbf{R}$ is finitary if it is bounded and continuous with respect to this topology and with the real line given the discrete topology. A subset of $H$ is called finitary if its indicator function is a finitary function. Dubins and Savage showed in [6] that a strategy, $\sigma$, induces a finitely additive probability measure upon the algebra of finitary sets. Thus, identifying $\sigma$ with this measure, if $g$ is a finitary function then $\int g d \sigma$ is defined. In this paper, we want to work with a larger class of functions than the class of finitary functions. In particular, we will consider functions which are measurable with respect to the $\sigma$ algebra generated by the finitary sets. Denote this $\sigma$-algebra by $\Omega^{\infty}$. Purves and Sudderth in [15] (see also [5]) demonstrated that the measure induced by a strategy can be extended to a finitely additive measure on $\Omega^{\infty}$. Moreover, they extended the domain of definition of $\int g d \sigma$ (in such a way that certain important properties of the Dubins-Savage integral were retained) to all bounded functions which are measurable with respect to the $\sigma$-algebra generated by the open sets of the product discrete topology on $H$. We will identify a strategy with this extension. Furthermore, any time we mention a real valued $g$ defined on $H$ we will assume that it is bounded, positive, and $\Omega^{\infty}$-measurable.

The optimal return of a gambling problem is a real valued function, $V$, on $F, \mathrm{~d}$-sned by

$$
V(x)=\sup _{\sigma \in \Sigma(x)} \int g d \sigma .
$$

A strategy $\sigma$ available at $x$ is stationary if there exists a gamble valued function $S$ such that $S(x)=\sigma_{0}$ and for all partial histories $\left(x_{1}, \ldots, x_{n}\right)$, $\sigma_{n}\left(x_{1}, \ldots, x_{n}\right)=S\left(x_{n}\right)$.

A stationary plan $\widehat{S}$ is a function from $F$ into $P(F)$ whose graph lies in $\Gamma$ (i.e., $\widehat{S}(x) \in \Gamma(x)$ for all $x \in F$ ). Thus, for each $x \in F$, a stationary plan determines a stationary strategy, denoted by $\sigma^{\widehat{S}, x}$, where $\sigma_{0}^{\widehat{S}, x}=\widehat{S}(x)$ and $\left(\sigma^{\widehat{S}, x}\right)_{n}\left(x_{1}, \ldots, x_{n}\right)=\widehat{S}\left(x_{n}\right)$.

Stationary strategies are said to be adequate (for approximating optimal return) for the gambling problem $(F, \Gamma, g)$ if, for all $\varepsilon>0$ and all $x \in F$, there exists a stationary strategy $\sigma^{x} \in \Sigma(x)$ such that

$$
\int g d \sigma^{x} \geq V(x)-\varepsilon \text {. }
$$


Stationary plans are uniformly adequate if for each $\varepsilon>0$ there exists a stationary plane $\widehat{S}$ such that

$$
\int g d \sigma^{\widehat{S}, x} \geq V(x)-\varepsilon
$$

for all $x \in F$.

If $X$ is a Borel subset of a complete separable metric space then we denote by $B(X)$ the $\sigma$-algebra of Borel subset of $X . \widehat{P}(X)$ denotes the set of all countably additive probability measures defined on $(X, B(X)) . \widehat{P}(X)$ is endowed with the topology of weak convergence. Under this topology $\widehat{P}(X)$ is a Polish space and $B(\widehat{P}(X))$ is the smallest $\sigma$-algebra of sets which makes the maps $\rho \rightarrow \rho(A)$ measurable for each $A \in B(X)$ (see, for example, Parthasarathy [14, Chapter 2]).

Recall that a subset $A$ of $X$ is analytic if there exists a Borel space $Y$ and a Borel subset $C$ of $X \times Y$ such that $A$ is the projection of $C$ into $X$ (see [1, and 11] for a discussion of analytic sets). A real valued function, $f$, defined on $X$ is upper analytic if for every real number $r$, the sets $\{x \in X: f(x)>r\}$ are analytic. Analytic sets arise in gambling theory because often projections of Borel sets must be considered. And, as Lusin showed, these projections, analytic sets, are not necessarily Borel sets.

The universally measurable $\sigma$-algebra of subsets of $X, U(X)$, is that class defined by $U(X)=\bigcap_{\rho \in \widehat{P}(x)} B_{X}(\rho)$, where $B_{X}(\rho)$ is the completion of $B(X)$ with respect to $\rho$. A real valued function, $f$, defined on $X$ is universally measurable if it is measurable with respect to $(X, U(X))$ and $(\mathbf{R}, B(\mathbf{R}))$.

A gambling problem $(F, \Gamma, g)$ is analytic if:

(i) $F$ is a complete separable metric space.

(ii) $\Gamma$ is an analytic subset of $F \times \widehat{P}(F)$. In particular, every available gamble is countably additive when restricted to $B(F)$. Each such gamble is identified with its restriction.

(iii) $g$ is upper analytic on $H$ (recall that it is always assumed that $g$ is positive, bounded, and $\Omega^{\infty}$-measurable).

A gambling problem $(F, \Gamma, g)$ is leavable if $\delta_{x} \in \Gamma(x)$ for all $x \in F$, where $\delta_{x}$ is the unit mass at $x$. Essentially, this says the gambler can stop gambling at any time and go home with his present winnings.

Suppose that $g(h)=\lim \sup _{n \rightarrow \infty} u\left(x_{n}\right)$. Then it was shown in [3] that there exists a leavable Borel gambling problem (i.e., leavable analytic problem) for which stationary plans are not uniformly adequate. Stationary strategies, however, are adequate for $g(h)=\limsup _{n \rightarrow \infty} u\left(x_{n}\right)$ (see [19]). More generally, we get the following result.

Theorem 5.1. Let $(F, \Gamma, g)$ be a leavable analytic gambling problem. If $g$ is a shift invariant nearly leavable utility function then stationary strategies are adequate. 
The proof of Theorem 5.1 will be presented in $\S 5$. First, we define and characterize the class of shift invariant nearly leavable utility functions.

\section{NEARLY LEAVABLE, SHIFT INVARIANT UTILITY FUNCTIONS}

We begin this section with some definitions. A function $g: H \rightarrow \mathbf{R}$ is shift invariant if for every $h=\left(x_{1}, x_{2}, \ldots\right) \in H, g(h)=g\left(x_{2}, x_{3}, \ldots\right)$. A subset of $H$ is shift invariant if its indicator function is a shift invariant function.

A function $g: H \rightarrow \mathbf{R}$ is nearly leavable on a history $h=\left(x_{1}, x_{2}, \ldots\right) \in H$ if, for any $\varepsilon>0$, there exists an $N$ such that if $h^{\prime}=\left(x_{1}, x_{2}, \ldots, x_{N-1}, x_{N}\right.$, $\left.x_{N}, x_{N}, \ldots\right)$ then

$$
\left|g(h)-g\left(h^{\prime}\right)\right|<\varepsilon
$$

A function is nearly leavable if it is nearly leavable on all histories.

Suppose $g$ is shift invariant as well as nearly leavable. Then for any $\varepsilon>0$ and $h=\left(x_{1}, x_{2}, \ldots\right) \in H$, there exists a subsequence of coordinates of $h$, $\left\{x_{n_{k}}\right\}_{k=1}^{\infty}$, such that

$$
\left|g(h)-g\left(x_{n_{k}}, x_{n_{k}}, x_{n_{k}}, \ldots\right)\right|<\varepsilon \text { for } k=1,2, \ldots .
$$

Some examples of shift invariant nearly leavable functions include $u_{*}$ and the gambling function $u^{*}$, where $u_{*}(h)=\liminf _{n \rightarrow \infty} u\left(x_{n}\right)$ and $u^{*}(h)=$ lim $\sup _{n \rightarrow \infty} u\left(x_{n}\right)$ for some bounded function $u: F \rightarrow \mathbf{R}$. Other functions which at first glance do not appear to be shift invariant nearly leavable can be modified so as to fit into this setting. These functions include discounted total return, as studied by Blackwell in [2]. For instance, let $r$ be a bounded function of $F$, let $0<\beta<1$, and let $g: H \rightarrow \mathbf{R}$ be defined by $g(h)=\sum_{1}^{\infty} \beta^{n} r\left(x_{n}\right)$. In this form $g$ is neither shift invariant nor nearly leavable. Let us follow Example 2 of [18] and $\S 12.2$ of [6], and set $x_{n}^{\prime}=\left(x_{1}, \ldots, x_{n}\right)$ and $h^{\prime}=\left(x_{1}^{\prime}, x_{2}^{\prime}, \ldots\right)$. If we define $u: F^{\prime}=\bigcup_{n=1}^{\infty} F^{n} \rightarrow \mathbf{R}$ by $u\left(x_{n}^{\prime}\right)=\sum_{i=1}^{n} \beta^{i} r\left(x_{i}\right)$, then we have $u^{*}\left(h^{\prime}\right)=\limsup _{n \rightarrow \infty} u\left(x_{n}^{\prime}\right)=g(h)$, where $u^{*}$ is shift invariant and nearly leavable on $H^{\prime}$. (However, as our eventual goal is to demonstrate the adequacy of stationary strategies for shift invariant nearly leavable functions, it should be noted that a strategy which is stationary with respect to $H^{\prime}$ may not be stationary if translated back into a strategy on $H$.)

As suggested by $\mathrm{S}$. Ramakrishnan, it is possible to completely characterize all shift invariant nearly leavable functions. We shall do so with the following two propositions. Moreover, we shall demonstrate that this class of functions is closed under uniform limits.

For a subset $A$ of $F$, we denote by $\{A$ eventually $\}$ the set

$$
\left\{h=\left(x_{1}, x_{2}, \ldots\right) \in H: \text { there exists an } N(h) \text { such that } x_{j} \in A\right.
$$

$$
\text { for all } j \geq N(h)\},
$$

and we denote by $\{A$ i.o. $\}$ the set

$$
\left\{h=\left(x_{1}, x_{2}, \ldots\right) \in H \text { : for all } N \text { there exists a } j>N \text { such that } x_{j} \in A\right\} \text {. }
$$


Proposition 3.1. A function $g: H \rightarrow \mathbf{R}$, with finite range $\left\{a_{1}, \ldots, a_{n}\right\}$, is shift invariant nearly leavable if and only if there exists a partition $A_{1}, \ldots, A_{n}$ of $F$ and a partition $B_{1}, \ldots, B_{n}$ of $H$ such that

(i) $g$ is constant on each $B_{i}$,

(ii) each $B_{i}$ is shift invariant, and

(iii) $\left\{A_{i}\right.$ eventually $\} \subseteq B_{i} \subseteq\left\{A_{i}\right.$ i.o. $\}$ for each $i$.

Proof. To see the forward implication let $B_{i}=\left\{h: g(h)=a_{i}\right\}$, and let $A_{i}=$ $\left\{x \in F: g(x, x, x, x, \ldots)=a_{i}\right\}$. The other implication is easy to see.

Proposition 3.2. A bounded function $g: H \rightarrow \mathbf{R}$ is shift invariant nearly leavable if and only if it is the uniform limit of shift invariant nearly leavable functions with finite ranges.

Proof. To see this, suppose $g$ is a shift invariant nearly leavable function such that, for all $h, g(h)<\alpha<\infty$, for some constant $\alpha$ (we will without loss of generality assume $g$ is positive). For each positive integer $n$, and for each positive integer $k, 0 \leq k \leq n$, define the following sets:

$$
\begin{gathered}
M_{k, n}=\{h \in H: k \alpha / n<g(h)<(k+1) \alpha / n\}, \\
C_{k, n}=\{x \in F: k \alpha / n \leq g(x, x, x, \ldots) \leq(k+1) \alpha / n\}, \\
R_{k, n}=\left\{h \in H: g(h)=k \alpha / n, \text { and } h \in\left\{C_{k, n} i . o .\right\}\right\}, \\
L_{k, n}=\left\{h \in H: g(h)=k \alpha / n, \text { and } h \notin\left\{C_{k, n} i . o .\right\}\right\}, \\
B_{k, n}=R_{k, n} \cup M_{k, n} \cup L_{k+1, n},
\end{gathered}
$$

and define functions $f_{n}$ by

$$
f_{n}=\sum_{k=0}^{n}(k \alpha / n) I_{B_{k, n}} .
$$

Then the $f_{n}$ 's are shift invariant nearly leavable and their uniform limit is $g$. It is straightforward to show the other implication.

\section{TeChNiCAL RESUlts}

In this section we lay the groundwork needed to prove Theorem 5.1. Several of the results in this section provide generalizations of those found in $[8,6,19]$. We start with the following simple proposition.

Proposition 4.1. Let $\varepsilon>0, C \in \Omega^{\infty}$, and $\sigma^{1}, \sigma^{2}$ be strategies. Then there exists a finitary set $K$ such that $\sigma^{1}(C \triangle K) \leq \varepsilon$ and $\sigma^{2}(C \triangle K) \leq \varepsilon$.

Proof. If $C \in \Omega^{\infty}$ then by [15] there exists open sets $O_{1}, O_{2}$ such that $C \subseteq O_{i}$ and $\sigma^{i}\left(O_{i}-C\right)<\varepsilon / 2$ for $i=1,2$. Let $O=O_{1} \cap O_{2}$. 
By [5], there exist finitary sets, $K_{1}, K_{2}$, such that $K_{i} \subseteq O$ and $\sigma^{i}\left(O-K_{i}\right)<$ $\varepsilon / 2$ for $i=1,2$. Let $K=K_{1} \cup K_{2}$. Then $K$ is finitary and, for $i=1,2$,

$$
\begin{aligned}
\sigma^{i}(C \Delta K) & =\sigma^{i}(C-K)+\sigma^{i}(K-C) \\
& \leq \sigma^{i}(O-K)+\sigma^{i}(O-C) \leq \varepsilon
\end{aligned}
$$

Proposition 4.2 extends Proposition 4.1, and says that a $\Omega^{\infty}$-measurable function can be approximated by a finitary function, with respect to any two strategies.

Proposition 4.2. Given strategies $\sigma^{1}$ and $\sigma^{2}, \varepsilon>0$, and $g: H \rightarrow \mathbf{R}$, a positive bounded $\Omega^{\infty}$-measurable function, there exists a finitary function, $f$, such that for any $A \in \Omega^{\infty}$, and $i=1,2$,

$$
\left|\int_{A} g d \sigma^{i}-\int_{A} f d \sigma^{i}\right|<\varepsilon .
$$

Proof. If $g$ is a bounded $\Omega^{\infty}$-measurable function then there exists a $\Omega^{\infty}$ simple function, $s=\alpha_{1} I_{c_{1}}+\cdots+\alpha_{n} I_{c_{n}}$, such that, for all $h \in H$,

$$
|g(h)-s(h)|<\varepsilon / 2 \text {. }
$$

Moreover, for each $A \in \Omega^{\infty}$,

$$
\left|\int_{A} g d \sigma^{i}-\int_{A} s d \sigma^{i}\right|<\varepsilon / 2 .
$$

By Proposition 4.1, for each $j=1, \ldots, n$, there exist finitary sets $K_{j}$, such that, for $i=1,2$,

$$
\sigma^{i}\left(C_{j} \Delta K_{j}\right)<[\varepsilon /(4 M n)],
$$

where $M=\left|\alpha_{1}\right|+\cdots+\left|\alpha_{n}\right|$.

Define $f: H \rightarrow \mathbf{R}$ by $f=\alpha_{1} I_{K_{1}}+\cdots+\alpha_{n} I_{K_{n}}$. Then

$$
\begin{aligned}
\left|\int_{A} s d \sigma^{i}-\int_{A} f d \sigma^{i}\right| & \leq 2 M\left[\sigma^{i}(\{h: s(h) \neq f(h)\})\right] \\
& \leq 2 M\left[\sigma^{i}\left(\bigcup_{j=1}^{n}\left(C_{j} \Delta K_{j}\right)\right)\right] \\
& \leq 2 M\left[\sum_{j=1}^{n}\left(\sigma^{i}\left(C_{j} \Delta K_{j}\right)\right)\right] \leq \varepsilon / 2 .
\end{aligned}
$$

Thus,

$$
\left|\int_{A} g d \sigma^{i}-\int_{A} f d \sigma^{i}\right|<\varepsilon .
$$

A stopping time $t$ (called an incomplete stop rule in [6]) is a function from $H$ to the positive integers union $\{\infty\}$ such that, if $t(h)<\infty$ and $\hat{h}$ agrees with $h$ through the $t(h)$ coordinate, then $t(h)=t(\hat{h})$. 
Let $A$ be a finitary subset of $H$, and let $t$ be a stopping time that is everywhere less than infinity. $A$ is determined by time $t$ if for all $h, \hat{h} \in H$, which agree through the $t(h)$ coordinate, either both $h$ and $\hat{h}$ are in $A$ or both are not.

Frequent use will be made of the functions $p_{t}: H \rightarrow \bigcup_{n=1}^{\infty} F^{n}$, where $t$ is a stopping time. For $h=\left(x_{1}, x_{2}, \ldots\right)$ and $t(h)<\infty, p_{t}(h)=\left(x_{1}, \ldots, x_{t(h)}\right)$. For the special case of $t \equiv n, n$ a positive integer, the notation $p_{n}$ is used (note, in general, the functions $p_{t}$ are allowed to be undefined on some $h \in H$, that is, on $h$ such that $t(h)=\infty$ ).

The juxtaposition of a function $g$, defined on $H$, and a partial history, $p=\left(y_{1}, \ldots, y_{n}\right)$, denotes the function defined by

$$
g p(h)=g p\left(x_{1}, x_{2}, \ldots\right)=g\left(y_{1}, \ldots, y_{n}, x_{1}, x_{2}, \ldots\right) \text {. }
$$

Propositions 4.3 and 4.4 explicitly state two results which are fairly immediate from [15].

Proposition 4.3. Let $g$ be a positive bounded $\Omega^{\infty}$-measurable function, and let $A$ be a finitary subset determined by time $t$, where $t$ is an everywhere finite stopping time. Then

$$
\int_{A} g d \sigma=\int_{A} \int\left(g p_{t}(h)\right) d \sigma\left[p_{t}(h)\right] d \sigma(h) .
$$

Proof. Using results from $[15,16]$ (specifically, Corollary 4.1 and Theorem 5.1 of [15], and $\S 9$ of [16]) it is straightforward to show that

$$
\int_{A} g d \sigma=\iint\left(I_{A} \cdot g\right) p_{t}(h) d \sigma\left[p_{t}(h)\right] d \sigma(h) .
$$

If $A$ is determined by time $t$, then, for $h \in H, I_{A} p_{t}(h)\left(h^{\prime}\right)=I_{A}(h)$, for all $h^{\prime} \in H$. The result now follows.

Proposition 4.4. If $g$ is a positive bounded $\Omega^{\infty}$-measurable function and $\tau$ is a stopping time, then

(i) $\int_{[\tau<\infty]} g d \sigma=\sup _{t} \int_{[\tau \leq t]} g d \sigma$,

(ii) $\int_{[\tau=\infty]}^{[\tau<\infty} g d \sigma=\inf _{t} \int_{[\tau>t]}^{[\tau \leq l]} g d \sigma$,

where the supremum and infimum are taken over all everywhere finite stopping times.

Proof. Immediate result of Corollary 5.3 of [15].

Let $\sigma$ and $\hat{\sigma}$ be strategies and let $t$ be a stopping time. If for all $h \in H$ and $n<t(h) \sigma\left[p_{n}(h)\right]=\hat{\sigma}\left[p_{n}(h)\right]$, then $\sigma$ agrees with $\hat{\sigma}$ up to time $t$. In this case, Lemma 4.1 states that the difference in the utilities of the two strategies is completely determined by their conditional strategies after time $t$, provided that the utility function is shift invariant. This result generalizes Lemma 3 of [8], where the utility function was assumed to be $u^{*}$. 
Lemma 4.1. Let $\tau$ be a stopping time, let $\sigma^{1}$ and $\sigma^{2}$ be strategies which agree up to time $\tau$, and let $g$ be a positive, bounded, shift invariant, $\Omega^{\infty}$-measurable function. If for all $h \in H$ such that $\tau(h)<\infty$,

$$
\int g d \sigma^{1}\left[p_{\tau}(h)\right] \geq \int g d \sigma^{2}\left[p_{\tau}(h)\right]-\varepsilon,
$$

then $\int g d \sigma^{1} \geq \int g d \sigma^{2}-\varepsilon$.

Proof. Let $t$ be an everywhere finite stopping time and let $s=\tau \wedge t$. The set $[\tau \leq t]$ is determined by time $s$. By Proposition 4.3, then,

$$
\begin{aligned}
\int_{[\tau \leq t]} g d \sigma^{i} & =\int_{[\tau \leq t]} \int g p_{s}(h) d \sigma^{i}\left[p_{s}(h)\right] d \sigma^{i}(h) \\
& =\int_{[\tau \leq t]} \int g d \sigma^{i}\left[p_{s}(h)\right] d \sigma^{i}(h)
\end{aligned}
$$

for $i=1,2$. If $h \in[\tau \leq t]$ then $s(h)=\tau(h)$, and so

$$
\int g d \sigma^{1}\left[p_{s}(h)\right] \geq \int g d \sigma^{2}\left[p_{s}(h)\right]-\varepsilon .
$$

Therefore, for any everywhere finite stopping time, $t$,

$$
\int_{[\tau \leq t]} g d \sigma^{1} \geq \int_{[\tau \leq t]} g d \sigma^{2}-\varepsilon
$$

From Proposition 4.4 we get

$$
\int_{[\tau<\infty]} g d \sigma^{1} \geq \int_{[\tau<\infty]} g d \sigma^{2}-\varepsilon
$$

Now, by Proposition 4.4, there exists an everywhere finite stopping time, $t$, and, by Proposition 4.2, there is a finitary function, $f$, such that for any $A \in \Omega^{\infty}$ and $i=1,2$,

$$
\left|\int_{[\tau>t]} g d \sigma^{i}-\int_{[\tau=\infty]} g d \sigma^{i}\right|<\varepsilon / 4
$$

and

$$
\left|\int_{A} g d \sigma^{i}-\int_{A} f d \sigma^{i}\right|<\varepsilon / 4
$$

Let $r$ be the time by which $f$ is determined, let $w=r \vee t$, and let $z=\tau \wedge w$. Then $\sigma^{1}$ and $\sigma^{2}$ agree up to time $z$, and the function $f \cdot I_{[\tau>w]}$ is determined by time $z$. Lemma 2 of [8] then implies

$$
\int_{[\tau>w]} f d \sigma^{1}=\int f I_{[\tau>w]} d \sigma^{1}=\int f I_{[\tau>w]} d \sigma^{2}=\int_{[\tau>w]} f d \sigma^{2}
$$


Therefore,

$$
\begin{aligned}
& \left|\int_{[\tau=\infty]} g d \sigma^{1}-\int_{[\tau=\infty]} g \sigma^{2}\right| \\
& \leq\left|\int_{[\tau=\infty]} g d \sigma^{1}-\int_{[\tau>w]} g d \sigma^{1}\right|+\left|\int_{[\tau>w]} g d \sigma^{1}-\int_{[\tau>w]} f d \sigma^{1}\right| \\
& \quad+\left|\int_{[\tau>w]} f d \sigma^{1}-\int_{[\tau>w]} f \sigma^{2}\right|+\left|\int_{[\tau>w]} f d \sigma^{2}-\int_{[\tau>w]} g d \sigma^{2}\right| \\
& \quad+\left|\int_{[\tau>w]} g d \sigma^{2}-\int_{[\tau=\infty]} g d \sigma^{2}\right| \\
& \leq \varepsilon .
\end{aligned}
$$

This inequality follows from the above constructions, and the obvious fact that if $t_{1} \geq t_{2}$, then for any $\sigma$,

$$
\left|\int_{[\tau=\infty]} g d \sigma-\int_{\left[\tau>t_{1}\right]} g d \sigma\right| \leq\left|\int_{[\tau=\infty]} g d \sigma-\int_{\left[\tau>t_{2}\right]} g d \sigma\right| .
$$

Since $\varepsilon$ was arbitrary,

$$
\int_{[\tau=\infty]} g d \sigma^{1}=\int_{[\tau=\infty]} g d \sigma^{2}
$$

The lemma now follows from (1) and (2).

A strategy, $\sigma$, stagnates immediately at $x \in F$ if $\sigma=\delta\left(h_{x}\right)$, where $h_{x}=$ $(x, x, x, \ldots)$ and $\delta\left(h_{x}\right)$ is the unit mass at $h_{x}$. A strategy stagnates along $a$ history $h=\left(x_{1}, x_{2}, \ldots\right)$ at time $n$ if $\sigma\left[p_{n}(h)\right]=\delta\left(h_{x_{n}}\right)$.

For a strategy $\sigma$, we define a stopping time $s_{\sigma}$ by letting $s_{\sigma}(h)$ be the first $n$, if any, for which $\sigma$ stagnates along $h$ at time $n$. If $\sigma$ does not stagnate along $h$ for any $n$, then $s_{\sigma}(h)=\infty$. This stopping time is called the stagnation time of $\sigma$. With these definitions let

$$
S(x)=\left\{\sigma \in \Sigma(x): s_{\sigma}(h)<\infty \text { for all } h \in H\right\},
$$

and

$$
S^{n}(x)=\left\{\sigma \in \Sigma(x): s_{\sigma}(h) \leq n \text { for all } h \in H\right\} .
$$

For $g: H \rightarrow \mathbf{R}$, a positive bounded $\Omega^{\infty}$-measurable function, the following functions are various measures of how well a gambler can do if his gambling time is limited:

$$
\begin{gathered}
U_{0}(x)=g\left(h_{x}\right), \quad U_{n}(x)=\sup _{\sigma \in S^{n}(x)} \int g d \sigma, \\
U_{\omega}(x)=\lim _{n \rightarrow \infty} U_{n}(x), \quad U(x)=\sup _{\sigma \in S(x)} \int g d \sigma .
\end{gathered}
$$

Theorem 4.1 is our first gambling result involving nearly leavable shift invariant utility functions. It states that a gambler may do just as well by using stagnating strategies as he can by using all strategies.

A state $x \in F$ is $\varepsilon$-adequate if $g\left(h_{x}\right) \geq V(x)-\varepsilon$. 
Theorem 4.1. Suppose $(F, \Gamma, g)$ is a leavable gambling problem. If $g$ is a nearly leavable shift invariant utility function (as usual, we assume $g$ is positive, bounded and $\Omega^{\infty}$-measurable), then $U=V$.

Proof. Clearly $V \geq U$. Now let $\varepsilon>0$ be given and choose $\sigma \in \Sigma(x)$ such that $\int g d \sigma>V(x)-\varepsilon^{2}$. Let $\tau$ be the hitting time of the $2 \varepsilon$-adequate elements of $F$. Then,

$$
\left\{h: V^{*}(h)-g(h)<\varepsilon\right\} \subseteq\{h: \tau(h)<\infty\} .
$$

To see this note that if $\hat{h}=\left(x_{1}, x_{2}, \ldots\right)$ is not in $\{h: \tau(h)<\infty\}$ then, for all $n$, we have $V\left(x_{n}\right)>g\left(h_{x_{n}}\right)+2 \varepsilon$. But, since $g$ is shift invariant nearly leavable, there exists, $x_{n_{1}}, x_{n_{2}}, \ldots$, coordinates of $\hat{h}$, such that $\left|g(\hat{h})-g\left(h_{x_{n_{k}}}\right)\right|<\varepsilon$ for all $k$, and so, $V\left(x_{n_{k}}\right)>g(\hat{h})+\varepsilon$ for all $k \geq 1$. This implies $V^{*}(\hat{h})>g(\hat{h})+\varepsilon$. That is, $\hat{h}$ is not in $\left\{h: V^{*}(h)-g(h)<\varepsilon\right\}$. By Theorems 7 and 13 of [18], $\sigma\{h: \tau(h)<\infty\}>1-\varepsilon$.

Define a strategy $\sigma^{\prime}$ such that $\sigma^{\prime}$ agrees with $\sigma$ up to time $\tau$, and if $\tau(\hat{h})<$ $\infty$ then $\sigma^{\prime}\left[p_{\tau}(\hat{h})\right]=\delta\left(h_{x_{\tau}(\hat{h})}\right)$. That is, $\sigma^{\prime}$ agrees with $\sigma$ until a $2 \varepsilon$-adequate fortune is hit and then $\sigma^{\prime}$ stagnates.

Notice, that for all histories $h=\left(x_{1}, x_{2}, \ldots\right)$ for which $\tau(h)<\infty$,

$$
\int g d \sigma^{\prime}\left[p_{\tau}(h)\right]>V\left(x_{\tau(h)}\right)-2 \varepsilon>\int g d \sigma\left[p_{\tau}(h)\right]-2 \varepsilon .
$$

Thus, by Lemma 4.1, $\int g d \sigma^{\prime}>\int g d \sigma-2 \varepsilon$. Furthermore, $\sigma^{\prime}\{h: \tau(h)=\infty\}=$ $\sigma\{h: \tau(h)=\infty\}$ (this follows from the proof of Lemma 4.1).

Select an everywhere finite stopping time, $t$, such that

$$
\int_{[\tau<\infty]} g d \sigma^{\prime}-\varepsilon<\int_{[\tau \leq t]} g d \sigma^{\prime}
$$

Now define a strategy $\sigma^{\prime \prime} \in S(x)$ such that $\sigma^{\prime \prime}$ agrees with $\sigma^{\prime}$ up to time $s=t \wedge \tau$, and then $\sigma^{\prime \prime}\left[p_{s}(\hat{h})\right]=\delta\left(h_{x_{s(h)}}\right)$.

Let

$$
m(h)=\int\left(g \cdot I_{[\tau \leq t]}\right) p_{s}(h) d \sigma^{\prime}\left[p_{s}(h)\right]
$$

and

$$
r(h)=\int\left(g \cdot I_{\tau \leq t]}\right) p_{s}(h) d \sigma^{\prime \prime}\left[p_{s}(h)\right]=\left(g \cdot I_{[\tau \leq t]}\right) p_{s}(h)\left(x_{s(h)}, x_{s(h)}, \ldots\right) .
$$

Note, first, that $m(h)=r(h)$ for all $h$; and second, $m$ and $r$ are determined by time $s$. Therefore, by Proposition 4.3 and Lemma 2 of [8],

$$
\int_{[\tau \leq t]} g d \sigma^{\prime}=\int m d \sigma^{\prime}=\int r d \sigma^{\prime \prime}=\int_{[\tau \leq t]} g d \sigma^{\prime \prime} .
$$


So we get

$$
\begin{aligned}
\int g d \sigma^{\prime \prime} & =\int_{[\tau \leq t]} g d \sigma^{\prime \prime}+\int_{[\tau>t]} g d \sigma^{\prime \prime} \\
& =\int_{[\tau \leq t]} g d \sigma^{\prime}+\int_{[\tau>t]} g d \sigma^{\prime \prime} \geq \int_{[\tau<\infty]} g d \sigma^{\prime}-\varepsilon \\
& \geq \int g d \sigma^{\prime}-\varepsilon M-\varepsilon \quad\left(\text { where } M=\sup _{h \in H}|g(h)|\right) \\
& \geq \int g d \sigma-2 \varepsilon-\varepsilon M-\varepsilon \geq V(x)-\varepsilon^{2}-2 \varepsilon-\varepsilon M-\varepsilon .
\end{aligned}
$$

Thus, since $\sigma^{\prime \prime} \in S(x)$ and $\varepsilon$ was arbitrary, we have $U=V$.

The following propositions generalize results found in $[6,19]$ to the case of bounded, shift invariant, $\Omega^{\infty}$-measurable utility functions.

Proposition 4.5. Suppose $(F, \Gamma, g)$ is a leavable gambling problem, and let $g$ be a shift invariant utility function. Then $U_{n+1}(x)=\sup _{\gamma \in \Gamma(x)} \int U_{n} d \gamma$.

Proof. Let $\varepsilon>0$. Choose $\sigma \in S^{n+1}(x)$ such that $\int g d \sigma \geq U_{n+1}(x)-\varepsilon$. Clearly, for all $y \in F, \sigma[y] \in S^{n}(y)$. Thus,

$$
\begin{aligned}
U_{n+1}(x)-\varepsilon & \leq \int g d \sigma=\iint g d \sigma[y] d \sigma_{0} \\
& \leq \int U_{n}(y) d \sigma_{0} \leq \sup _{\gamma \in \Gamma(x)} \int_{U_{n}} d \gamma .
\end{aligned}
$$

Since $\varepsilon$ was arbitrary, $U_{n+1}(x) \leq \sup _{\gamma \in \Gamma(x)} \int U_{n} d \gamma$.

For the other inequality, again, let $\varepsilon>0$, and let $\gamma \in \Gamma(x)$. For each $y \in F$ choose a $\sigma^{y} \in S^{n}(y)$ such that $U_{n}(y)-\varepsilon \leq \int g d \sigma^{y}$. Define a strategy, $\sigma$, in $S^{n+1}(x)$ by $\sigma_{0}=\gamma$ and $\sigma[y]=\sigma^{y}$. Then

$$
\begin{aligned}
U_{n+1}(x) & \geq \int g d \sigma=\iint g d \sigma[y] d \sigma_{0} \\
& \geq \int U_{n} d \gamma-\varepsilon .
\end{aligned}
$$

Lemma 4.2 gives a reformulation of Lemma 2 of [19] to the shift invariant case. It, as well as Proposition 4.5, are used in conjunction with other results of [19] to prove Proposition 4.6.

Lemma 4.2. Suppose $(F, \Gamma, g)$ is a leavable gambling problem. Let $\alpha>0$, let $g$ be shift invariant, and let $W: F \rightarrow \mathbf{R}$ be a bounded function. Suppose $\sigma$ is a strategy available at $x \in F$ such that, either $g\left(h_{x_{n}}\right)=W\left(x_{n}\right)$ and $\sigma$ stagnates at time $n$ along $\left(x_{1}, \ldots, x_{n}, \ldots\right)$ or $\int W d \sigma\left(x_{1}, \ldots, x_{n}\right) \geq W\left(x_{n}\right)+\alpha$. Then $\int g d \sigma \geq \int W^{*} d \sigma \geq W(x)$ and $\sigma\left[s_{\sigma}<n\right] \rightarrow 1$ as $n \rightarrow \infty$.

Proof. The fact that $\sigma\left[s_{\sigma}<n\right] \rightarrow 1$ as $n \rightarrow \infty$ follows from the first part of Lemma 2 of [19]. 
For each positive integer $n$, let $t_{n}=s_{\sigma} \wedge n$. The sets $\left[s_{\sigma} \geq n\right]$ and $\left[s_{\sigma}<n\right]$, are, then, determined by time $t_{n}$. Thus,

$$
\begin{aligned}
\int\left(W^{*}-g\right) d \sigma= & \int_{\left[s_{\sigma} \geq n\right]} \int\left(W^{*}-g\right) p_{t_{n}}(h) d \sigma\left[p_{t_{n}}(h)\right] d \sigma(h) \\
& +\int_{\left[s_{\sigma}<n\right]} \int\left(W^{*}-g\right) p_{t_{n}}(h) d \sigma\left[p_{t_{n}}(h)\right] d \sigma(h) \\
= & \int_{\left[s_{\sigma} \geq n\right]}\left(W^{*}-g\right) d \sigma .
\end{aligned}
$$

The first equality follows from Proposition 4.3, and the second holds by the hypotheses and because $\left(W^{*}-g\right)$ is shift invariant.

Recall that if $\widehat{S}$ is a stationary plan, then $\sigma^{\widehat{S}, x}$ denotes the stationary strategy available at $x$ determined by $\widehat{S}$.

Proposition 4.6. Let $(F, \Gamma, g)$ be a leavable gambling problem in which $g$ is shift invariant and let $\varepsilon>0$. Then for each positive integer, $n$, there exists a stationary plan $\widehat{S}_{n}$ available in $(F, \Gamma, g)$ such that for every $x \in F$,

$$
\int g d \sigma^{\widehat{S}_{n}, x} \geq U_{n}(x)-\varepsilon .
$$

Proof. Apply an easy adaptation of the first part of Theorem 1 of [19] to the shift invariant case, then finish the proof using Lemma 4.2 and Proposition 4.5 .

The next result is the penultimate step in proving the adequacy of stationary strategies for shift invariant nearly leavable utility functions. The corollary states that stationary strategies are adequate if $U_{\omega}=U$. In the following section this is shown to be true if the gambling problem is analytic.

Corollary 4.1. Let $(F, \Gamma, g)$ be a leavable gambling problem, and suppose $g$ is shift invariant nearly leavable. If $U_{\omega}=U$ then for each $x \in F$ and $\varepsilon>0$ there exists a stationary strategy, $\sigma$, available at $x$, such that $\int g d \sigma \geq V(x)-\varepsilon$.

Proof. Apply Proposition 4.6 and Theorem 4.1.

\section{ADEQUACY OF STATIONARY STRATEGIES}

The results of the last section were obtained within a very general setting. In particular, no measurability assumptions were made concerning the state space. In this section, for the first time, we require that the gambling problem by analytic. The section culminates with the proof of Theorem 5.1.

Suppose $(F, \Gamma, g)$ is an analytic gambling problem. Let $A(F)$ be the $\sigma$ algebra generated by the analytic subsets of $F$. A strategy, $\sigma$, available in this gambling problem is analytically measurable if, for all $n$ and $A \in A(F)$, the maps

$$
\left(x_{1}, \ldots, x_{n}\right) \rightarrow \sigma_{n}\left(x_{1}, \ldots, x_{n}\right)(A)
$$


are measurable from $\left(F^{n},(A(F))^{n}\right)$ to $(\mathbf{R}, B(\mathbf{R}))$, where $B(\mathbf{R})$ are the Borel subsets of $\mathbf{R}$.

An analytically measurable strategy, $\sigma$, determines a countably additive measure, $\mu(\sigma)$, on $(H, B(H))$, where $B(H)$ is the sigma field $B(F) \times B(F) \times$ $B(F) \times \cdots$ of Borel subsets of $H$. That is, the $\mu(\sigma)$ marginal distribution of $x_{1}$ is $\sigma_{0}$, and for every $n \geq 1$ and $\left(x_{1}, \ldots, x_{n}\right)$, the $\mu(\sigma)$ conditional distribution of $x_{n+1}$ given $\left(x_{1}, \ldots, x_{n}\right)$ is $\sigma_{n}\left(x_{1}, \ldots, x_{n}\right)$. Note that if $C \in B_{H}(\mu(\sigma))$ and $C \in \Omega^{\infty}$, then $\sigma(C)=\mu(\sigma)(C)$ (see Theorem 6.1 and the remark after the proof of the theorem in [15]). With some ambiguity, we write $\sigma$ for $\mu(\sigma)$.

The set of all analytically measurable strategies available at $x \in F$ is denoted by $\Sigma^{A}(x)$. Let

$$
\begin{gathered}
\Sigma^{A}=\left\{(x, \sigma): x \in F, \sigma \in \Sigma^{A}(x)\right\}, \\
\Sigma_{n}^{A}(x)=\left\{\sigma \in \Sigma^{A}(x): \sigma \text { stagnates by time } n \text { with } \sigma \text { probability } 1\right\},
\end{gathered}
$$

and

$$
\Sigma_{n}^{A}=\left\{(x, \sigma): x \in F, \sigma \in \Sigma_{n}^{A}(x)\right\} .
$$

The sets $\Sigma^{A}$ and $\Sigma_{n}^{A}$ are analytic subsets of $F \times \widehat{P}(H)$, where $\widehat{P}(H)$ is the set of countably additive probability measures on $(H, B(H))$. The fact that $\Sigma^{A}$ is analytic follows from Theorem 3 of [4]. To see that $\Sigma_{n}^{A}$ is analytic, note that Theorem 3 of [4] can be used to show that the set $\Psi_{n}$, where

$$
\begin{aligned}
\Psi_{n}=\{(x, \nu): & x \in F, \\
\nu & \text { is analytically measurable } n \text {-day strategy available at } x\},
\end{aligned}
$$

is an analytic subset of $F \times \widehat{P}\left(F^{n}\right)$. If $\sigma$ defined by the sequence $\sigma_{0}, \sigma_{1}, \ldots$ is an analytically measurable strategy available at $x \in F$, then the finite sequence $\sigma_{0}, \ldots, \sigma_{n-1}$ is an analytically measurable $n$-day strategy available at $x$. An analytically measurable $n$-day strategy determines a countably additive measure on $\left(F^{n}, B\left(F^{n}\right)\right)$. It can be shown that $\Sigma_{n}^{A}$ is an analytic embedding of $\Psi_{n}$ in $\Sigma^{A}$. Alternately, using Proposition 1.1 of [12] it is routine to modify Theorem 2.1 of [19] to show that $\Sigma^{A}$ and $\Sigma_{n}^{A}$ are analytic.

Define the function $Q_{n}$ by

$$
Q_{n}(x)=\sup _{\sigma \in \Sigma_{n}^{A}(x)} \int g d \sigma .
$$

We now proceed to show that $U_{\omega}=U$.

Proposition 5.1. Let $(F, \Gamma, g)$ be a leavable analytic gambling problem in which $g$ is shift invariant. Then, for each $n \geq 1, U_{n}$ is a universally measurable function.

Proof. As mentioned, $\Sigma_{n}^{A}$ is an analytic subset of $F \times \widehat{P}(H)$. Therefore, $\left\{(x, \sigma) \in \Sigma_{n}^{A}: \int g d \sigma>r\right\}$ is analytic, and so the projection of this set, $\left\{x: Q_{n}(x)>r\right\}$, is analytic. That is, $Q_{n}$ is upper analytic. Now adapt the proof of Theorem 2.2 of [19] to finish the proof of this more general case. 
A real valued function, $W$, defined on $F$ is excessive with respect to a gambling problem $(F, \Gamma, g)$, if for all $x \in F$ and for each $\gamma \in \Gamma(x), \int W d \gamma \leq$ $W(x)$.

Proposition 5.2. Let $(F, \Gamma, g)$ be a leavable analytic gambling problem, and suppose that $g$ is shift invariant. Then $U_{\omega}$ is excessive with respect to $(F, \Gamma, g)$. Proof. Since the $U_{n}$ are universally measurable, for any $\gamma$ available in $(F, \Gamma$, g),

$$
\lim _{n \rightarrow \infty} \int U_{n} d \gamma=\int U_{\omega} d \gamma
$$

Thus,

$$
\int U_{\omega} d \gamma \leq \lim _{n \rightarrow \infty} U_{n+1}(x)=U_{\omega}(x)
$$

Let $\pi(x)=\{(\sigma, t): \sigma \in \Sigma(x)$, and $t$ is an everywhere finite stopping time $\}$. For a real valued function, $G$, defined on $F$, let $G((\sigma, t))=\int G\left(f_{t}\right) d \sigma$, where $f_{t}(h)=x_{t(h)}$ for $h=\left(x_{1}, x_{2}, \ldots\right)$.

The next proposition has Theorem 2.12.1 of [6] as a predecessor.

Proposition 5.3. Let $(F, \Gamma, g)$ be a leavable gambling problem and suppose $g$ is shift invariant. Suppose $Q: F \rightarrow \mathbf{R}$ is excessive with respect to $(F, \Gamma, g)$ and for all $x \in F, Q(x) \geq \hat{g}(x)$, where $\hat{g}: F \rightarrow \mathbf{R}$ is defined by $\hat{g}(x)=g\left(h_{x}\right)$. then $Q \geq U$.

Proof. If $Q$ is excessive with respect to $(F, \Gamma, g)$ then, by Lemma 2.12 .2 of [6], $Q(\sigma, t) \leq Q(x)$ for all $x \in F$, and $(\sigma, t) \in \Pi(x)$. Since $\hat{g} \leq Q$, for all $\pi=(\sigma, t) \in \Pi(x)$, we have $\hat{g}(\pi) \leq Q(\pi) \leq Q(x)$. Now, if $\sigma \in S(x)$ let $\pi_{\sigma}=\left(\sigma, s_{\sigma}\right)$. Then, since $G$ is shift invariant, $\hat{g}\left(\pi_{\sigma}\right)=\int g d \sigma$. And thus, $U(x) \leq Q(x)$.

Now, armed with the above results, the proof of Theorem 5.1 becomes trivial.

Theorem 5.1. Let $(F, \Gamma, g)$ be a leavable analytic gambling problem, and suppose, additionally, that $g$ is a nearly leavable shift invariant. Then stationary strategies are adequate for approximating optimal return.

Proof. Apply Propositions 5.2 and 5.3, and Corollary 4.1.

\section{NECESSITY OF NEARLY LEAVABILITY, SHIFT INVARIANCE}

Ostensibly, the question which should now be asked is whether it is necessary for the utility function to be nearly leavable and shift invariant in order for stationary strategies to be adequate. However, a moment's reflection indicates that, perhaps, this is not the appropriate question after all. Specifically, no matter what properties the utility function has or does not have it is possible to construct $\Gamma$ so that stationary strategies are adequate. This can be done by simply having just one gamble available at each point in the state space. Therefore, it seems that we should ask, given a utility function which is not 
nearly leavable shift invariant, does there exist some $\Gamma$ for which stationary strategies are not adequate? Again, unfortunately, this does not appear to be the case. We do, however get Proposition 6.1.

A history $h^{\prime} \in H$ is eventually constant if there exists an $x \in F$ such that $h^{\prime}=\left(p, h_{x}\right)$, where $p$ is a finite sequence of fortunes. Let $H_{c}$ denote the set of eventually constant histories. Proposition 6.1 indicates that $g$ must be nearly leavable shift invariant on some histories, other than those in $H_{c}$, in order for stationary strategies to be adequate for every $\Gamma$.

Proposition 6.1. Let $F$ be a state space such that $|F| \geq 3$, and let $g$ be shift invariant. For all $h^{\prime}=\left(x_{1}^{\prime}, x_{2}^{\prime}, \ldots\right) \notin H_{c}$, suppose there exists an $\varepsilon\left(h^{\prime}\right)>0$ such that $g\left(h^{\prime}\right)>g\left(h_{x_{n}^{\prime}}\right)+\varepsilon\left(h^{\prime}\right)$ for all $n$. Then there exists a leavable gambling problem $(F, \Gamma, g)$ for which stationary strategies are not adequate.

Proof. Without loss of generality assume $F=\{x, y, z\}$ and $g\left(h_{z}\right) \geq g\left(h_{y}\right) \geq$ $g\left(h_{x}\right)$. Let $\hat{h}=(y, z, y, z, y, z, \ldots)$. Then, for some $\varepsilon>0, g(\hat{h}) \geq$ $g\left(h_{x}\right)+\varepsilon$.

Now define $\Gamma$ by

$$
\begin{gathered}
\Gamma(z)=\{\delta(z),(1 / n)(\delta(x))+(1-(1 / n))(\delta(y)), n=1,2, \ldots\}, \\
\Gamma(y)=\{\delta(y),(1 / n)(\delta(x))+(1-(1 / n))(\delta(z)), n=1,2, \ldots\}, \\
\Gamma(x)=\{\delta(x)\} .
\end{gathered}
$$

Thus, since $g$ is shift invariant, $\int g d \sigma=g\left(h_{x}\right)$ for any stationary strategy $\sigma \in \Sigma(z)$. But, clearly

$$
V(z) \geq g(\hat{h}) \geq g\left(h_{x}\right)+\varepsilon .
$$

Acknowledgment. The author wishes to thank the referee for his careful reading of the manuscript and his useful suggestions.

\section{REFERENCES}

1. D. P. Bertsekas and S. E. Shreve, Stochastic optimal control: The discrete time case, Academic Press, New York, 1978.

2. David Blackwell, Discounted dynamic programming, Ann Math. Statist. 36 (1965), 226235.

3. D. Blackwell and S. Ramakrishnan, Stationary plans need not be uniformly adequate for leavable Borel gambling problems, Proc. Amer. Math. Soc. 102 (1988), 1024-1027.

4. Claude Dellacherie, Quelques resultats sur les maisons de jeux analytiques (Seminaire de Probabilites XIX, Univ. Strasbourg), Lecture Notes in Math., vol. 1123, Springer, 1985, pp. 221-229.

5. L. E. Dubins, On Lebesgue-like extensions of finitely additive measures, Ann. Probab. 2 (1974), 456-463.

6. L. E. Dubins and L. J. Savage, How to gamble if you must, McGraw-Hill, New York, 1965.

7. L. E. Dubins and W. D. Sudderth, An example in which stationary strategies are not adequate, Ann. Probab. 3 (1975), 722-725.

8. __ Persistently E-optimal strategies, Math. Oper. Res. 2 (1977), 125-134. 
9. T. P. Hill, On the existence of good Markov strategies, Trans. Amer. Math. Soc. 247 (1979), 157-176.

10. T. P. Hill and V. C. Pestien, The existence of good Markov strategies for decision processes with general payoffs, Stochastic Process Appl. 24 (1988), 61-76.

11. K. Kuratowski and A. Mostowski, Set theory, Polish Scientific Publishers, Warsaw, 1976.

12. Michael Monticino, The adequacy of measurable and of stationary strategies for approximating optimal return, Ph.D. thesis, Univ. of Miami, 1987.

13. Donald Ornstein, On the existence of stationary optimal strategies, Proc. Amer. Math. Soc. 20 (1969), 563-569.

14. K. R. Parthasarathy, Probability measures on metric spaces, Academic Press, New York, 1967.

15. R. A. Purves and W. D. Sudderth, Some finitely additive probability, Ann. Probab. 4 (1976), 259-276.

16. __ Some finitely additive probability, University of Minnesota Technical Report No. 220, 1973.

17. W. D. Sudderth, Gambling problems with limit inferior payoff, Math. Oper. Res. 8 (1983), 287-297.

18. _ On the Dubins and Savage characterization of optimal strategies, Ann. Math. Statist. 43 (1972), 498-507.

19. $\ldots$, On the existence of good stationary strategies, Trans. Amer. Math. Soc. 135 (1969), 399-414.

Daniel H. Wagner, Associates, 894 Ross Drive, Suite 205, Sunnyvale, California 94089

Current address: Department of Mathematics, University of North Texas, Denton, Texas 76203 